Annu Rev Physiol. 2010 March 17; 72: 81-94. doi:10.1146/annurev-physiol-021909-135931.

\title{
The FoxO Family in Cardiac Function and Dysfunction
}

\author{
Sarah M. Ronnebaum and \\ McAllister Heart Institute, University of North Carolina, Chapel Hill, North Carolina 27599; \\ sarah.ronnebaum@unc.edu \\ Cam Patterson * \\ McAllister Heart Institute, University of North Carolina, Chapel Hill, North Carolina 27599; \\ cpatters@med.unc.edu
}

\begin{abstract}
The Forkhead family of transcription factors mediates many aspects of physiology, including stress response, metabolism, commitment to apoptosis, and development. The Forkhead box subfamily $\mathrm{O}$ (FoxO) proteins have garnered particular interest due to their involvement in the modulation of cardiovascular biology. In this review, we discuss the mechanisms of FoxO regulation and outcomes of FoxO signaling under normal and pathological cardiovascular contexts.
\end{abstract}

\section{Keywords}

Forkhead; Akt signaling; stress response; cardiac hypertrophy

\section{INTRODUCTION}

The Forkhead/winged helix family of transcription factors, characterized by a conserved DNA binding domain known as the Forkhead box (Fox), is essential in both development and adult physiology. First described in 1989 (1), further identification of related Forkhead proteins has led to the characterization of 19 subfamilies, designated A-S (reviewed in 2,3). Several Forkhead proteins, including FoxC1, FoxC2, FoxH1, FoxM1, FoxO1, and FoxP1, appear to be essential for normal cardiac development, as their deletion in mice results in severe arterial and cardiac defects and early lethality (reviewed in 4). In addition, an abundance of evidence suggests that three members of the FoxO subfamily-FoxO1, FoxO3, and FoxO4-are critical in maintaining cardiac function and mediating cardiac stresses in the adult (the remaining FoxO family member, FoxO6, is localized in the brain). Within the setting of cardiac function, the FoxO family is believed to be involved in diverse activities, including response to oxidative stress (5), regulation of metabolism (6), cell cycle control (7), and commitment to apoptosis (8). This review focuses primarily on the regulation and outcomes of FoxO signaling in the heart.

Copyright () 2010 by Annual Reviews. All rights reserved

*Corresponding author..

DISCLOSURE STATEMENT

The authors are not aware of any affiliations, memberships, funding, or financial holdings that might be perceived as affecting the objectivity of this review. 


\section{FOXO TRANSCRIPTIONAL ACTIVITY}

In the nucleus, FoxO proteins target a conserved DNA binding sequence, TTGTTTAC (9), and can associate with coactivators such as Smad (10), Notch (11), $\beta$-catenin (12), and PGC-1 $\alpha$ (6). The end result of FoxO transcriptional activity is generally associated with counteracting oxidative stress and promoting cell cycle arrest and apoptosis. Early experiments studying FoxO function demonstrated that overexpression of constitutively active FoxO1 causes apoptosis, and this outcome is dependent on FoxO-DNA binding (13). This proapoptotic function is mediated through transcription of several target genes, including Fas (14), Bim (15), TRAIL (16), and Puma (17). In addition, FoxO causes cell cycle arrest via transcription of $p 27^{k i p 1}(8), p 21^{c i p l}(10), p 130$, and the inhibitory cyclin $G 2$ (18). Conversely, FoxO proteins are also involved in the promotion of DNA repair through increased expression of several genes, including Gadd45a (19). In addition, the antioxidant enzymes MnSOD (manganese superoxide dismutase) and catalase are FoxO target genes (5), and transcription of these enzymes is induced by FoxO to counteract oxidative stress. FoxO can activate the synthesis of PEPCK (phosphoenolpyruvate carboxykinase) and glucose-6-phophatase, two enzymes necessary for gluconeogenesis (20). Lastly, FoxO can mediate the stress response by interfering with other transcription factors to inhibit transcription (21-23).

\section{MECHANISMS OF FOXO REGULATION}

\section{FoxO and Akt Signaling}

The FoxO subfamily members were originally identified by their involvement in chromosomal translocations associated with leukemias and rhabdomyosarcomas $(24,25)$. Concurrent work in Caenorhabditis elegans demonstrated a link between hormonal inputs, Akt signaling, and FoxO. In C. elegans, inactivating mutations in daf-2 (homolog of the insulin receptor) or age-1 [homolog of phosphoinositide-3 kinase (PI3K)] cause growth arrest and life span extension; however, these effects are dependent on the activity of the FoxO homolog daf-16 $(26,27)$. This observation led to the discovery that FoxO transcriptional activity is inhibited by PI3K-Akt signaling (28) whereby PI3K phosphorylation causes Akt to translocate to the nucleus (29), where it phosphorylates FoxO (Thr24, Ser256, Ser319 of FoxO1; Thr32, Ser253, Ser315 of FoxO3). FoxO contains a nuclear export sequence and a nuclear localization sequence, the latter of which is masked following phosphorylation by Akt $(30,31)$. Phosphorylation by Akt promotes FoxO interaction with a subset of 14-3-3 proteins localized in the nucleus, causing translocation of the FoxO-14-3-3 complex to the cytoplasm via the nuclear export protein Crm1. Once in the cytosol, FoxO is then sequestered by 14-3-3 proteins and is rendered inactive $(14,32)$. Mutation of the Akt phosphorylation sites on FoxO proteins results in exclusive FoxO nuclear localization and insusceptibility to Akt-mediated inhibition (33). Cumulatively, PI3K-Akt signaling is a major regulator of FoxO activity.

\section{Additional FoxO Regulation}

In addition to phosphorylation and inhibition by Akt, recent work suggests that FoxO is also regulated by several posttranslational modifications, including phosphorylation by multiple kinases, glycosylation, acetylation, and ubiquitination. The details of these regulatory pathways are described below.

Phosphorylation and inhibition-In addition to Akt, SGK1 (serum and glucocorticoidinduced kinase 1) can phosphorylate and inhibit FoxO3 activity through nuclear exclusion (34). Although Akt and SGK1 share FoxO phosphorylation sites, SGK1 favors phosphorylation of Ser315, whereas Akt most actively phosphorylates Ser253. Under some 
stimuli, coordinate activities of these proteins may synergistically inhibit FoxO3 function. Like Akt, SGK1 is activated by the PI3K signaling cascade and has many overlapping functions with Akt. However, in the case of FoxO3 at least, the regulation by SGK1 and Akt appears to be a nonredundant, complementary relationship. For example, radiation-induced DNA damage stimulates p53 expression, which inhibits FoxO3 through an SGK1dependent, but not an Akt-dependent, manner (35). In addition, Wnt activation and $\beta$-catenin accumulation lead to an upregulation of SGK1 and subsequent inhibition of FoxO3 activity (36).

CDK2 (cyclin-dependent kinase 2) promotes G1/S cell cycle progression by associating with cyclin E and cyclin A. CDK2 can phosphorylate Ser249 and Ser298 of FoxO1 to cause cytoplasmic retention and inhibit FoxO1 activity (37). However, under DNA-damaging conditions, CDK2 activity is inhibited by the FoxO target genes $p 21^{\text {cip } 1}$ and $p 27^{k i p 1}$ (38), allowing FoxO1 to activate target gene expression, thereby forming a positive feedback loop.

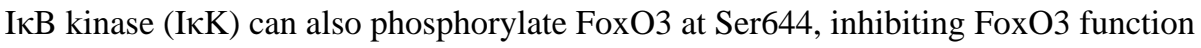
independently of Akt (39). In addition to promoting cytoplasmic retention of FoxO3, IкK mediates the polyubiquitination and degradation of FoxO3 via the proteosome system. Similarly, ERK (extracellular signal-regulated protein kinase) phosphorylation of FoxO3 (at Ser 294, Ser 344, and Ser 425) in response to EGF stimulation also increases cytosolic FoxO3 levels and promotes interaction and degradation by the E3 ubiquitin ligase MDM2 (mouse double minute 2) (40).

FoxO1, FoxO3, and FoxO4 share a highly conserved serine-rich domain (Ser319, Ser322, Ser325, Ser329 of FoxO1), suggestive of regulation by kinase signaling pathways. The phosphorylation of Ser319 by Akt or SGK1 is required for CK1 (casein kinase 1)-dependent Ser322 and Ser325 phosphorylation (41). Furthermore, Ser329 is phosphorylated by DYRK1A (dual-specificity tyrosine phosphorylated and regulated kinase 1A) (42). Together, phosphorylation of these residues provides a strong signal promoting nuclear exclusion.

Phosphorylation and activation-FoxO3 can be directly phosphorylated by AMPK (AMP-activated protein kinase), an energy-sensing protein activated when ATP levels are low (43). However, unlike Akt phosphorylation, which results in FoxO cytoplasmic retention and inhibition of FoxO target gene transcription, AMPK phosphorylation increases FoxO3 target gene transcription without affecting FoxO3 subcellular localization. The observation that Akt phosphorylation and AMPK phosphorylation produce opposite outcomes on FoxO activity can be attributed to the fact that Akt does not share any of the six unique AMPK phosphorylation sites identified in vitro on FoxO3, of which three (Ser413, Ser588, Ser626) are functional in vivo. Furthermore, the signals leading to Akt or AMPK activation generally occur under the opposite circumstances of energy abundance versus energy conservation, respectively. Although it is not currently known whether FoxO1 is also activated by AMPK, in vitro assays have demonstrated that FoxO1 is phosphorylated by AMPK (44).

A second kinase capable of promoting FoxO activation is JNK (c- $\underline{\mathrm{J} u n}-\underline{\mathrm{N}}$-terminal kinase). Oxidative stress induced by hydrogen peroxide exposure leads to activation of the GTPase Ral and subsequent activation of JNK, which can in turn phosphorylate FoxO4 on two Cterminal residues (Thr447 and Thr451) to promote nuclear translocation and activation of FoxO4 gene transcription (45). JNK-dependent activation of FoxO in response to reactive oxygen species induces expression of the FoxO target genes encoding MnSOD and catalase to form an efficient feedback loop. Interestingly, although JNK-dependent FoxO activation 
in response to stress is conserved in Drosophila and C. elegans, the sites of JNK phosphorylation are not conserved in either species. Instead, the Drosophila homolog dFOXO (46), and the C. elegans homolog daf-16 are phosphorylated by JNK in their N termini (47). The JNK target sites on FoxO4 are also not conserved in FoxO1 or FoxO3, but evidence suggests that FoxO1 and FoxO3 are also activated, either by direct phosphorylation or by inhibition of the FoxO-retaining protein $14-3-3$, in a JNK-dependent manner $(48,49)$.

Oxidative stress also induces autophosphorylation and activation of MST1 (mammalian sterile twenty 1), a member of a kinase family involved in stress response and JNK activation. Activated MST1 interacts with the forkhead domain of FoxO3 to directly phosphorylate a conserved serine residue (Ser207), which reduces FoxO interaction with 14-3-3 proteins and initiates nuclear translocation and target gene transcription (50). Overexpression of the C. elegans MST1 homolog cst- 1 increases longevity in a daf-16dependent manner. Although MST1 and JNK participate in similar signaling pathways, JNK is unable to phosphorylate the forkhead domain, suggesting that MST1 and JNK may act in a complementary fashion to activate FoxOs following oxidative stress. Although these studies demonstrate the capability of MST1 to phosphorylate FoxO1 in vitro, subsequent in vitro work has shown that MST1 does not phosphorylate DNA-bound FoxO1 (51). In fact, MST1-phosphorylated FoxO1 has greatly diminished DNA binding affinity (52). Although it appears that MST1-mediated FoxO phosphorylation promotes nuclear translocation, the subsequent dephosphorylation of FoxO may be required for transcriptional activity. Incidentally, Akt suppresses MST1-mediated FoxO3 phosphorylation, demonstrating an indirect mechanism of FoxO suppression by Akt (53).

Glycosylation-Another mechanism of FoxO regulation is mediated through addition of $\mathrm{N}$-acetylglucosamine (O-GlcNAc) by O-GlcNAc transferase (OGT), a dynamic modification of serine/threonine residues similar to phosphorylation. Generally, this modification is highly sensitive to glucose concentrations and cell stress, and several proteins involved in glucose metabolism, oxidative stress, and mitosis, including transcription factors, are OGlcNAcylated (reviewed in 54). FoxO1 O-GlcNAcylation by OGT increases transcription of some FoxO target genes independent of Akt phosphorylation (55,56). In addition, PGC-1 $\alpha$, previously shown to be a FoxO coactivator and now shown to also be O-GlcNAcylated, recruits OGT to FoxO1 to increase FoxO activation (57).

Acetylation-Early studies suggested acetylation-dependent FoxO regulation when it was demonstrated that the $\mathrm{p} 300 / \mathrm{CBP}$ coactivator complex, which possesses histone acetyltransferase (HAT) activity, interacts with FoxO1, and inhibition of this interaction reduces FoxO transcriptional activity (58). However, subsequent examinations of the effects of $\mathrm{p} 300 / \mathrm{CBP}$ acetylation have been conflicting. A current model suggests that $\mathrm{p} 300$-induced FoxO1 acetylation promotes FoxO1-mediated gene transcription in the absence of insulin (59). Conversely, insulin receptor signaling increases FoxO1 acetylation dependent on Aktmediated phosphorylation but does not induce FoxO1 transcriptional activity. In contrast, CBP acetylates a region of the DNA-binding domains of FoxO1, FoxO3, and FoxO4 (Lys242, Lys245, Lys262 on FoxO1), resulting in decreased DNA binding affinity, reduced transcriptional activity, and increased susceptibility to Akt-mediated phosphorylation at $\operatorname{Ser} 253(60,61)$.

The NAD-dependent histone deacetylase SirT1 (silent mating type information regulation 2 homolog 1) targets many substrates, particularly proteins involved in metabolism and stress response (reviewed in 62). In C. elegans, caloric restriction-dependent life span extension via overexpression of the SirTl homolog sir-2 is dependent on daf-16 (63), suggesting a relationship between the human homologs $\mathrm{SirTl}$ and FoxO. FoxO3 and SirT1 loosely interact under unstressed conditions, but following hydrogen peroxide exposure or heat 
shock, FoxO3 is highly acetylated and tightly interacts with SirT1 (64). The SirT1-FoxO interaction represents a complicated biology that remains to be elucidated. SirT1 deacetylates p300 and inhibits p300-dependent increases in FoxO transcription of Bim (65). However, SirT1 enhances transcription of some FoxO target genes (66), suggesting an overall model in which SirT1 increases the ability of FoxO to respond to stress through cell cycle arrest and other adaptations but inhibits FoxO transcription of apoptotic genes. In addition, evidence suggests that SirT1 deacetylation increases FoxO polyubiquitination and degradation (67).

Ubiquitination-Following activation of Akt, both FoxO1 and FoxO3 are degraded by the proteosome (68); however, the ubiquitination of FoxO1 and FoxO3 is mediated through different pathways. FoxO1 is degraded by Skp2, an F-box protein and subunit of Skp1/Cul1/ F-box ubiquitin ligase complex (69). FoxO1 ubiquitination by Skp2 is dependent on Akt phosphorylation of Ser256, providing an additional level of FoxO regulation by Akt. In addition, Skp2 also ubiquitinates and degrades p2 $7^{\mathrm{kip} 1}$ (7). However, Skp2 does not appear to interact with FoxO3. Instead, FoxO3 appears to be polyubiquitinated and degraded via MDM2, which is promoted by phosphorylation of FoxO3 by ERK (40), as discussed above. FoxO3 may also be degraded through cleavage by caspase-3-like proteases (70).

Ubiquitin-dependent degradation of FoxO4 has not been identified; however, following hydrogen peroxide treatment, MDM2 monoubiquitinates FoxO4 to increase nuclear localization and transcriptional activity $(71,72)$. This modification is reversed by the ubiquitin-specific protease USP7, which deubiquitinates FoxO4 and causes nuclear export.

The FoxO target gene product atrogin-1 ubiquitinates and degrades calcineurin, preventing Akt-mediated hypertrophic signaling. In addition, atrogin- 1 also acts as a FoxO coactivator through noncanonical polyubiquitination, which serves to increase FoxO activity and oppose Akt-mediated signaling (73). Atrogin-1 does not tag FoxO with canonical polyubiquitin chains that lead to proteosomal degradation and does not appear to directly affect FoxO stability. The consequences of atrogin-1-dependent ubiquitination are discussed in more detail below.

\section{FOXO AND CARDIAC BIOLOGY}

\section{Cardiac Development}

Despite some of the overlapping effects of the FoxO proteins, studies involving the deletion of individual FoxO family members suggest that each of the FoxOs has very different roles in vascular and cardiac development. For instance, whereas FoxO3 ${ }^{-/-}$and $\mathrm{FoxO}^{-/-}$ embryos develop normally (74), FoxO1 ${ }^{-1-}$ embryos die by day E10.5-E11 and exhibit grossly deficient vascular and cardiac growth $(74,75)$. FoxO1 is expressed in embryonic vessels, so it is not surprising that FoxO1 $1^{-1-}$ embryos exhibit impaired yolk sac vasculature, underdeveloped dorsal aorta, and impaired cardiac looping. Interestingly, though, $\mathrm{FoxO}^{+/-}$ mice develop normally. Conversely, mice overexpressing a cardiac-specific FoxO1 transgene also die by E10.5 due to abnormal $\mathrm{p} 21^{\text {cip } 1}$ and $\mathrm{p} 27^{\mathrm{kip} 1}$ expression, resulting in impaired cardiomyocyte proliferation, reduced heart size and myocardium thickness, and subsequent heart failure (76).

FoxO proteins are also involved in the regulation of vascular development. FoxO3 represses

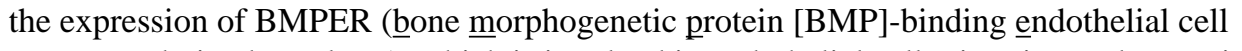
precursor-derived regulator), which is involved in endothelial cell migration and sprouting during development $(77,78)$. Consistent with these data, FoxO1 or FoxO3 overexpression in HUVEC cells decreases endothelial cell sprouting and migration; conversely, silencing 
FoxO1 or FoxO3 promotes angiogenesis (79). Additionally, negative regulation of FoxO1 by SirT1 appears to be important for supporting sprout formation (80).

The enhancer region of myocardin, which acts as a coactivator to induce smooth muscle cell and cardiomyocyte differentiation, contains FoxO binding sites and requires FoxO for its activity (81). FoxOs also maintain normal endothelial cell physiology in the adult, as demonstrated by mice engineered with floxed FoxO alleles that develop hemangiomas with advancing age (82).

\section{Cardiac Hypertrophy}

Maintaining FoxO activity is essential in preventing Akt-mediated cardiac hypertrophy. The IGF-Akt signaling cascade promotes cardiac hypertrophy by increasing cell size and protein translation via activation of S6K and mTOR and by decreasing antihypertrophic transcriptional activity via inhibition of GSK3 (reviewed in 83). By inhibiting FoxO activity, Akt also suppresses transcription of two key FoxO target gene products, the muscle-specific ubiquitin ligases atrogin-1/MAFbx and MuRF1 (84). The cumulative effect of Akt activation in the heart is an increase in progrowth pathways and the inhibition of FoxOmediated catabolism, resulting in hypertrophy. The CIRKO (cardiac insulin receptor knockout) mouse, which displays a reduction in cardiac mass due to reduced cardiomyocyte size (85), demonstrates the necessity of Akt signaling in cardiac hypertrophy. The phenotype of the CIRKO mouse is mimicked in mice with heart-specific FoxO3 expression (86). Furthermore, adenovirus-mediated FoxO3 overexpression reduces growth factor- and stretch-induced hypertrophy in cultured cardiomyocytes (86). In contrast, heart size is doubled in the heart-specific Akt transgenic mouse (87), consistent with evidence that $\mathrm{FoxO}^{-1-}$ mice demonstrate an increase in cardiac mass (88).

In addition to Akt signaling, a second major pathway involved in cardiac hypertrophy is mediated by the calcium-regulated protein phosphatase calcineurin (or PP2B). Calcineurin dephosphorylates the transcription factor NFAT (nuclear factor of activated $\underline{T}$ cells), which promotes NFAT nuclear translocation and induces transcription of genes characteristic of the hypertrophic response such as those encoding $\alpha$-skeletal actin and $\beta$-myosin heavy chain (89). Akt, FoxO, atrogin-1, and calcineurin were first connected in cardiomyocytes with the discovery that atrogin-1 targets calcineurin for degradation, decreases NFAT-dependent transcription, and prevents calcineurin-dependent cardiac hypertrophy (90), suggesting that FoxO might regulate calcineurin-NFAT signaling. Accordingly, overexpression of FoxO in cultured cardiomyocytes increases atrogin- 1 expression, inhibits calcineurin activity, and decreases angiotensin II-induced cardiac hypertrophy (88). Atrogin-1 overexpression decreases Akt-mediated FoxO1/3 phosphorylation, and furthermore, transgenic mice with cardiomyocyte-restricted overexpression of atrogin-1 are resistant to Akt-dependent hypertrophy (73). Although it was previously shown that the phosphatase activity of PP2A inhibits Akt (91), it has since been shown that calcineurin also dephosphorylates and inactivates Akt and that FoxO attenuates the interaction between PP2A and Akt (92). In summary, when FoxO is activated, FoxO-dependent atrogin-1 transcription leads to decreased calcineurin levels and, accordingly, decreased phosphatase activity directed toward Akt. Furthermore, because FoxO inhibits the interaction between Akt and PP2A, the net result is hyperphosphorylation of Akt (Figure 1). Surprisingly, the FoxO-induced increase in Akt phosphorylation appears to preferentially cause FoxO phosphorylation, and not phosphorylation of the Akt targets mTOR and GSK3. Thus, FoxO activity is controlled through a negative feedback loop. 


\section{Cardiac Stress Response and Aging}

The outcome of FoxO signaling in different contexts can appear contradictory. For example, although FoxOs increase antioxidant enzymes to counter oxidative stress, FoxOs also promote apoptotic signaling pathways under other circumstances. The effects of FoxOs on aging and stress response in the heart and vasculature involve a complex relationship between many signaling pathways.

As mentioned above, an antiaging role of forkhead proteins is supported by the evidence of increased longevity in C. elegans with active daf-16. FoxOs also have an antiaging function in the heart, first exhibited by the prevention of cardiac decline by transgenic expression in Drosophila (93). One major pathway through which FoxO signaling prevents cardiac aging is via activation of SirT1, which has a conserved role in preventing an aging phenotype in many tissues (reviewed in 62). SirT1 is upregulated by pressure overload, and cardiacspecific overexpression of SirT1 helps prevent oxidative stress and aging via FoxO1dependent expression of catalase (94). Additionally, moderate SirT1 transgenic expression diminishes age-related heart hypertrophy; in contrast, a transgenic mouse line demonstrating $\sim 12$-fold SirT1 overexpression demonstrates left ventricular fibrosis, apoptosis, and hypertrophy-associated fetal gene expression, suggestive of dose-dependent SirT1 effects. Finally, activation of SirT1 via resveratrol treatment in a cardiomyocyte cell line inhibits hypoxia-induced apoptosis (95). These effects appear to be mediated through decreased expression of the proapoptotic FoxO target Bim and increased expression of the FoxO1 target $\mathrm{p} 27^{\mathrm{kip} 1}$, which causes cell cycle arrest. Incidentally, it has been demonstrated that the inhibition of hypertrophy by resveratrol treatment is mediated through activation of AMPK, which, as described above, can also promote FoxO activity (96).

In addition to stimulating the antioxidant activities of MnSOD and catalase, FoxO3 also regulates transcription of a mitochondrial hydrogen peroxide scavenger, peroxiredoxin III, in cardiac fibroblasts (97). Peroxiredoxin III expression prevents serum withdrawal-induced apoptosis and may have an important role as a cardioprotectant in vivo.

The endothelial cell stress response is partially mediated by VEGF (vascular endothelial growth factor), which promotes angiogenesis through endothelial cell survival, migration, and proliferation. VEGF and FoxO have a complicated relationship in endothelial cells. VEGF induces NADPH oxidase activity, which activates a redox-sensitive Akt signaling pathway and mediates some effects of VEGF. VEGF also causes transient phosphorylation and nuclear exclusion of FoxO1, FoxO3, and FoxO4 via NADPH oxidase-dependent Akt signaling, which prevents apoptosis, promotes cell cycle progression, and downregulates expression of most FoxO1 target genes $(98,99)$. However, a small subset of FoxO target genes (including those encoding MnSOD, BMP2, VCAM-1, and MMP10) is upregulated by VEGF in a FoxO1-dependent manner (100). Unlike most FoxO1 target genes, expression of this subset is not immediately inhibited by VEGF, and transcription of these genes may be aided by NF-kB or NFAT. Despite the fact that VEGF generally inhibits FoxO activity, the VEGF-dependent activation of a subset of FoxO1 target genes helps explain why FoxO1 $1^{-/-}$ mice demonstrate an impaired vasculature phenotype.

In the vasculature, Akt is the major antagonist of FoxO signaling. Under some stress conditions, FoxO inhibition is beneficial to maintaining vessel viability. Shear stress, heat shock, and anoxia induce Akt signaling and subsequently inhibit FoxO, which promotes cell survival, evasion of apoptosis, and early senescence (101-103).

\section{Cardiac Metabolism and Autophagy}

Through a variety of transcriptional targets, FoxOs can respond to a changing metabolic environment via regulation of metabolic enzymes and energy-dependent proteins. The direct 
metabolic effects of FoxO signaling are not entirely clear; however, because FoxOs may be involved in transcription of many metabolic genes, a bioinformatics approach may help uncover FoxO-regulated genes. In Drosophila, a significant portion of genes upregulated by fasting-refeeding are also activated in cells expressing constitutively active $d F O X O$ (104). In humans, mice, and rats, FoxO binding sites are found in the promoters of several metabolic genes, including those encoding acetyl CoA carboxylase 2, the fatty acid transporter CD36, lipoprotein lipase, and Glut4 (105). FoxO proteins may also indirectly exert metabolic effects through transcription of PGC- $1 \alpha$, which associates with PPAR $\gamma$ to activate genes involved in mitochondrial biogenesis, fatty acid oxidation, and gluconeogenesis $(66,106)$. Additionally, PGC- $1 \alpha$ can serve as a FoxO coactivator to regulate expression of metabolic genes. For example, in hepatocytes, PGC-1 $\alpha$ and FoxO1 associate with estrogen-related receptors to activate transcription of pyruvate dehydrogenase kinase 4 , which limits the production of acetyl CoA from pyruvate and suppresses glucose oxidation in the absence of insulin (107).

FoxOs also regulate expression of genes that sense changes in the metabolic environment. For example, FoxO1 and FoxO3 activate transcription of Kir6.2, an ATP-dependent potassium channel ( $\mathrm{K}_{\mathrm{ATP}}$ ) subunit (105). In the presence of ATP, $\mathrm{K}_{\mathrm{ATP}}$ channels close, causing membrane depolarization and calcium influx, thereby maintaining contractile function. Under metabolically stressful conditions, ATP levels are low and $\mathrm{K}_{\mathrm{ATP}}$ channels remain open, such that the cell is hyperpolarized and calcium demand is limited. Transgenic mice lacking Kir6.2 demonstrate severe heart failure within hours of transaortic banding (108).

FoxO also provides a balance between metabolic pathways and protein degradation. In addition to regulating the transcription of the E3 ligases atrogin-1 and MuRF1, FoxO can promote proteolysis through autophagy. Autophagy recaptures amino acids and fatty acids for ATP production and new protein translation (reviewed in 109). In cardiomyocytes, autophagy is activated during ischemia-reperfusion and transaortic constriction-induced heart failure. Accordingly, increased autophagy reduces apoptosis following ischemiareperfusion (110), whereas acute cardiac-specific inhibition of autophagy causes left ventricular dilation, decreased contractile function, and hypertrophy (111). In myocytes, FoxO3 regulates transcription of autophagy genes, such as those encoding Gabarapl1, Atg12l, and LCB3, and promotes autophagosome formation (112). Thus, FoxOs promote proteolysis through activation of the ubiquitin ligases MuRF1 and atrogin-1 and through increased transcription of genes necessary for autophagy.

\section{CONCLUDING REMARKS}

The Forkhead subfamily $\mathrm{O}$ is an important mediator of the stress response. First characterized for its necessity for longevity in C. elegans, it has since been demonstrated that FoxOs have unexpected and diverse roles in countering stress, determining cell fate, and regulating energy availability. Because the heart is constantly adapting to different stresses and metabolic conditions, FoxOs appear to play an extremely important role in cardiac physiology. As we learn more precise information about FoxO regulation, direct transcriptional targets, and dynamic signaling responses, a therapeutic role for FoxOs may be elucidated. The rapidly growing field of forkhead biology will surely provide information and models of how cells grow, change, and adapt.

\section{SUMMARY POINTS}

1. FoxO proteins mediate stress response through transcription of genes involved in oxidative stress, metabolism, autophagy, aging, and apoptosis. 
2. FoxO proteins are regulated by a variety of mechanisms, including phosphorylation, glycosylation, acetylation, and ubiquitination. These modifications can activate or inhibit FoxO activity.

3. In cardiomyocytes, regulation of FoxO signaling is complex and inhibited by negative feedback. Akt inhibits FoxO activity while promoting NFAT-mediated hypertrophy, whereas overexpression of FoxO leads to eventual Akt hyperphosphorylation and FoxO inhibition. FoxO overexpression prevents Aktdependent cardiac hypertrophy.

4. FoxO helps mediate the antiaging effects of SirT1 through increased transcription of antioxidant and cell cycle arrest proteins while inhibiting transcription of FoxO proapoptotic targets.

\section{Acknowledgments}

We thank Andrea Portbury for helpful discussion. This work was supported by National Institutes of Health grants GM61728, HL65619, and AG024282 to C.P.

\section{Glossary}

FoxO

MnSOD

PI3K

PI3K-Akt signaling

SirT1

Cardiac

hypertrophy

MuRF1

NFAT

Autophagy
Forkhead box subfamily O

manganese superoxide dismutase

phosphoinositide-3 kinase

activated by insulin receptor. Akt activity leads to increased glucose uptake, cell cycle progression, evasion of apoptosis, and protein translation

silent mating type information regulation 2 homolog 1

increased cell size in cardiomyocytes in response to stress or growth factor stimulation

muscle ring finger 1

nuclear factor of activated $\mathrm{T}$ cells

catabolic lysosome-mediated protein degradation involved in energy and protein reallocation

\section{LITERATURE CITED}

1. Weigel D, Jurgens G, Kuttner F, Seifert E, Jackle H. The homeotic gene fork head encodes a nuclear protein and is expressed in the terminal regions of the Drosophila embryo. Cell 1989;57:645-58. [PubMed: 2566386]

2. Tuteja G, Kaestner KH. Forkhead transcription factors II. Cell 2007;131:192. [PubMed: 17923097]

3. Tuteja G, Kaestner KH. SnapShot: forkhead transcription factors I. Cell 2007;130:1160. [PubMed: 17889656]

4. Papanicolaou KN, Izumiya Y, Walsh K. Forkhead transcription factors and cardiovascular biology. Circ. Res 2008;102:16-31. [PubMed: 18174472]

5. Kops GJ, Dansen TB, Polderman PE, Saarloos I, Wirtz KW, et al. Forkhead transcription factor FOXO3a protects quiescent cells from oxidative stress. Nature 2002;419:316-21. [PubMed: 12239572] 
6. Puigserver P, Rhee J, Donovan J, Walkey CJ, Yoon JC, et al. Insulin-regulated hepatic gluconeogenesis through FOXO1-PGC-1 $\alpha$ interaction. Nature 2003;423:550-55. [PubMed: 12754525]

7. Medema RH, Kops GJ, Bos JL, Burgering BM. AFX-like Forkhead transcription factors mediate cell-cycle regulation by Ras and PKB through p27 ${ }^{\text {kip1 }}$. Nature 2000;404:782-87. [PubMed: 10783894]

8. Stahl M, Dijkers PF, Kops GJ, Lens SM, Coffer PJ, et al. The forkhead transcription factor FoxO regulates transcription of $\mathrm{p} 7^{\mathrm{Kip} 1}$ and Bim in response to IL-2. J. Immunol 2002;168:5024-31. [PubMed: 11994454]

9. Furuyama T, Nakazawa T, Nakano I, Mori N. Identification of the differential distribution patterns of mRNAs and consensus binding sequences for mouse DAF-16 homologues. Biochem. J 2000;349:629-34. [PubMed: 10880363]

10. Seoane J, Le HV, Shen L, Anderson SA, Massagué J. Integration of Smad and forkhead pathways in the control of neuroepithelial and glioblastoma cell proliferation. Cell 2004;117:211-23. [PubMed: 15084259]

11. Kitamura T, Kitamura YI, Funahashi Y, Shawber CJ, Castrillon DH, et al. A Foxo/Notch pathway controls myogenic differentiation and fiber type specification. J. Clin. Investig 2007;117:2477-85. [PubMed: 17717603]

12. Essers MA, de Vries-Smits LM, Barker N, Polderman PE, Burgering BM, Korswagen HC. Functional interaction between $\beta$-catenin and FOXO in oxidative stress signaling. Science 2005;308:1181-84. [PubMed: 15905404]

13. Tang ED, Nuñez G, Barr FG, Guan KL. Negative regulation of the forkhead transcription factor FKHR by Akt. J. Biol. Chem 1999;274:16741-46. [PubMed: 10358014]

14. Brunet A, Bonni A, Zigmond MJ, Lin MZ, Juo P, et al. Akt promotes cell survival by phosphorylating and inhibiting a Forkhead transcription factor. Cell 1999;96:857-68. [PubMed: 10102273]

15. Dijkers PF, Medema RH, Lammers JW, Koenderman L, Coffer PJ. Expression of the proapoptotic Bcl-2 family member Bim is regulated by the forkhead transcription factor FKHR-L1. Curr. Biol 2000;10:1201-4. [PubMed: 11050388]

16. Modur V, Nagarajan R, Evers BM, Milbrandt J. FOXO proteins regulate tumor necrosis factorrelated apoptosis inducing ligand expression. Implications for PTEN mutation in prostate cancer. J. Biol. Chem 2002;277:47928-37. [PubMed: 12351634]

17. You H, Pellegrini M, Tsuchihara K, Yamamoto K, Hacker G, et al. FOXO3a-dependent regulation of Puma in response to cytokine/growth factor withdrawal. J. Exp. Med 2006;203:1657-63. [PubMed: 16801400]

18. Chen J, Yusuf I, Andersen HM, Fruman DA. FOXO transcription factors cooperate with $\delta \mathrm{EF} 1$ to activate growth suppressive genes in B lymphocytes. J. Immunol 2006;176:2711-21. [PubMed: 16493026]

19. Tran H, Brunet A, Grenier JM, Datta SR, Fornace AJ Jr, et al. DNA repair pathway stimulated by the forkhead transcription factor FOXO3a through the Gadd45 protein. Science 2002;296:530-34. [PubMed: 11964479]

20. Nakae J, Kitamura T, Silver DL, Accili D. The forkhead transcription factor Foxo1 (Fkhr) confers insulin sensitivity onto glucose-6-phosphatase expression. J. Clin. Investig 2001;108:1359-67. [PubMed: 11696581]

21. Dowell P, Otto TC, Adi S, Lane MD. Convergence of peroxisome proliferator-activated receptor $\gamma$ and Foxo1 signaling pathways. J. Biol. Chem 2003;278:45485-91. [PubMed: 12966085]

22. Hirota K, Daitoku H, Matsuzaki H, Araya N, Yamagata K, et al. Hepatocyte nuclear factor-4 is a novel downstream target of insulin via FKHR as a signal-regulated transcriptional inhibitor. J. Biol. Chem 2003;278:13056-60. [PubMed: 12519792]

23. Zhao HH, Herrera RE, Coronado-Heinsohn E, Yang MC, Ludes-Meyers JH, et al. Forkhead homologue in rhabdomyosarcoma functions as a bifunctional nuclear receptor-interacting protein with both coactivator and corepressor functions. J. Biol. Chem 2001;276:27907-12. [PubMed: 11353774] 
24. Borkhardt A, Repp R, Haas OA, Leis T, Harbott J, et al. Cloning and characterization of $A F X$, the gene that fuses to MLL in acute leukemias with a $t(X ; 11)(q 13 ; q 23)$. Oncogene 1997;14:195-202. [PubMed: 9010221]

25. Galili N, Davis RJ, Fredericks WJ, Mukhopadhyay S, Rauscher FJ 3rd, et al. Fusion of a fork head domain gene to PAX3 in the solid tumour alveolar rhabdomyosarcoma. Nat. Genet 1993;5:230 35. [PubMed: 8275086]

26. Lin K, Dorman JB, Rodan A, Kenyon C. daf-16: an HNF-3/forkhead family member that can function to double the life-span of Caenorhabditis elegans. Science 1997;278:1319-22. [PubMed: 9360933]

27. Ogg S, Paradis S, Gottlieb S, Patterson GI, Lee L, et al. The Fork head transcription factor DAF-16 transduces insulin-like metabolic and longevity signals in C. elegans. Nature 1997;389:994-99. [PubMed: 9353126]

28. Kops GJ, de Ruiter ND, de Vries-Smits AM, Powell DR, Bos JL, Burgering BM. Direct control of the Forkhead transcription factor AFX by protein kinase B. Nature 1999;398:630-34. [PubMed: 10217147]

29. Meier R, Alessi DR, Cron P, Andjelkovic M, Hemmings BA. Mitogenic activation, phosphorylation, and nuclear translocation of protein kinase Bß. J. Biol. Chem 1997;272:3049197. [PubMed: 9374542]

30. Brownawell AM, Kops GJ, Macara IG, Burgering BM. Inhibition of nuclear import by protein kinase $\mathrm{B}$ (Akt) regulates the subcellular distribution and activity of the forkhead transcription factor AFX. Mol. Cell. Biol 2001;21:3534-46. [PubMed: 11313479]

31. Brunet A, Kanai F, Stehn J, Xu J, Sarbassova D, et al. 14-3-3 transits to the nucleus and participates in dynamic nucleocytoplasmic transport. J. Cell Biol 2002;156:817-28. [PubMed: 11864996]

32. Biggs WH 3rd, Meisenhelder J, Hunter T, Cavenee WK, Arden KC. Protein kinase B/Aktmediated phosphorylation promotes nuclear exclusion of the winged helix transcription factor FKHR1. Proc. Natl. Acad. Sci. USA 1999;96:7421-26. [PubMed: 10377430]

33. Takaishi H, Konishi H, Matsuzaki H, Ono Y, Shirai Y, et al. Regulation of nuclear translocation of forkhead transcription factor AFX by protein kinase B. Proc. Natl. Acad. Sci. USA 1999;96:11836-41. [PubMed: 10518537]

34. Brunet A, Park J, Tran H, Hu LS, Hemmings BA, Greenberg ME. Protein kinase SGK mediates survival signals by phosphorylating the forkhead transcription factor FKHRL1 (FOXO3a). Mol. Cell. Biol 2001;21:952-65. [PubMed: 11154281]

35. You H, Jang Y, You-Ten AI, Okada H, Liepa J, et al. p53-dependent inhibition of FKHRL1 in response to DNA damage through protein kinase SGK1. Proc. Natl. Acad. Sci. USA 2004;101:14057-62. [PubMed: 15383658]

36. Dehner M, Hadjihannas M, Weiske J, Huber O, Behrens J. Wnt signaling inhibits Forkhead box O3a-induced transcription and apoptosis through up-regulation of serum- and glucocorticoidinducible kinase 1. J. Biol. Chem 2008;283:19201-10. [PubMed: 18487207]

37. Huang H, Regan KM, Lou Z, Chen J, Tindall DJ. CDK2-dependent phosphorylation of FOXO1 as an apoptotic response to DNA damage. Science 2006;314:294-97. [PubMed: 17038621]

38. Harper JW, Elledge SJ, Keyomarsi K, Dynlacht B, Tsai LH, et al. Inhibition of cyclin-dependent kinases by p21. Mol. Biol. Cell 1995;6:387-400. [PubMed: 7626805]

39. Hu MC, Lee DF, Xia W, Golfman LS, Ou-Yang F, et al. IкB kinase promotes tumorigenesis through inhibition of forkhead FOXO3a. Cell 2004;117:225-37. [PubMed: 15084260]

40. Yang JY, Zong CS, Xia W, Yamaguchi H, Ding Q, et al. ERK promotes tumorigenesis by inhibiting FOXO3a via MDM2-mediated degradation. Nat. Cell Biol 2008;10:138-48. [PubMed: 18204439]

41. Rena G, Woods YL, Prescott AR, Peggie M, Unterman TG, et al. Two novel phosphorylation sites on FKHR that are critical for its nuclear exclusion. EMBO J 2002;21:2263-71. [PubMed: 11980723]

42. Woods YL, Rena G, Morrice N, Barthel A, Becker W, et al. The kinase DYRK1A phosphorylates the transcription factor FKHR at Ser329 in vitro, a novel in vivo phosphorylation site. Biochem. J 2001;355:597-607. [PubMed: 11311120] 
43. Greer EL, Oskoui PR, Banko MR, Maniar JM, Gygi MP, et al. The energy sensor AMP-activated protein kinase directly regulates the mammalian FOXO3 transcription factor. J. Biol. Chem 2007;282:30107-19. [PubMed: 17711846]

44. Dixit M, Bess E, Fisslthaler B, Hartel FV, Noll T, et al. Shear stress-induced activation of the AMP-activated protein kinase regulates FoxO1a and angiopoietin-2 in endothelial cells. Cardiovasc. Res 2008;77:160-68. [PubMed: 18006475]

45. Essers MA, Weijzen S, de Vries-Smits AM, Saarloos I, de Ruiter ND, et al. FOXO transcription factor activation by oxidative stress mediated by the small GTPase Ral and JNK. EMBO J 2004;23:4802-12. [PubMed: 15538382]

46. Wang MC, Bohmann D, Jasper H. JNK extends life span and limits growth by antagonizing cellular and organism-wide responses to insulin signaling. Cell 2005;121:115-25. [PubMed: 15820683]

47. Oh SW, Mukhopadhyay A, Svrzikapa N, Jiang F, Davis RJ, Tissenbaum HA. JNK regulates lifespan in Caenorhabditis elegans by modulating nuclear translocation of forkhead transcription factor/DAF-16. Proc. Natl. Acad. Sci. USA 2005;102:4494-99. [PubMed: 15767565]

48. Kawamori D, Kaneto H, Nakatani Y, Matsuoka TA, Matsuhisa M, et al. The forkhead transcription factor Foxo1 bridges the JNK pathway and the transcription factor PDX-1 through its intracellular translocation. J. Biol. Chem 2006;281:1091-98. [PubMed: 16282329]

49. Sunayama J, Tsuruta F, Masuyama N, Gotoh Y. JNK antagonizes Akt-mediated survival signals by phosphorylating 14-3-3. J. Cell Biol 2005;170:295-304. [PubMed: 16009721]

50. Lehtinen MK, Yuan Z, Boag PR, Yang Y, Villen J, et al. A conserved MST-FOXO signaling pathway mediates oxidative-stress responses and extends life span. Cell 2006;125:987-1001. [PubMed: 16751106]

51. Anand R, Kim AY, Brent M, Marmorstein R. Biochemical analysis of MST1 kinase: elucidation of a C-terminal regulatory region. Biochemistry 2008;42:6719-26. [PubMed: 18510339]

52. Brent MM, Anand R, Marmorstein R. Structural basis for DNA recognition by FoxO1 and its regulation by posttranslational modification. Structure 2008;16:1407-16. [PubMed: 18786403]

53. Jang SW, Yang SJ, Srinivasan S, Ye K. Akt phosphorylates MstI and prevents its proteolytic activation, blocking FOXO3 phosphorylation and nuclear translocation. J. Biol. Chem 2007;282:30836-44. [PubMed: 17726016]

54. Slawson C, Housley MP, Hart GW. O-GlcNAc cycling: how a single sugar post-translational modification is changing the way we think about signaling networks. J. Cell. Biochem 2006;97:71-83. [PubMed: 16237703]

55. Housley MP, Rodgers JT, Udeshi ND, Kelly TJ, Shabanowitz J, et al. O-GlcNAc regulates FoxO activation in response to glucose. J. Biol. Chem 2008;283:16283-92. [PubMed: 18420577]

56. Kuo M, Zilberfarb V, Gangneux N, Christeff N, Issad T. O-glycosylation of FoxO1 increases its transcriptional activity towards the glucose 6-phosphatase gene. FEBS Lett 2008;582:829-34. [PubMed: 18280254]

57. Housley MP, Udeshi ND, Rodgers JT, Shabanowitz J, Puigserver P, et al. A PGC-1 $\alpha$ :O-GlcNAc transferase complex regulates FoxO transcription factor activity in response to glucose. J. Biol. Chem 2008;284:5148-57. [PubMed: 19103600]

58. Nasrin N, Ogg S, Cahill CM, Biggs W, Nui S, et al. DAF-16 recruits the CREB-binding protein coactivator complex to the insulin-like growth factor binding protein 1 promoter in HepG2 cells. Proc. Natl. Acad. Sci. USA 2000;97:10412-17. [PubMed: 10973497]

59. Perrot V, Rechler MM. The coactivator p300 directly acetylates the forkhead transcription factor Foxo1 and stimulates Foxo1-induced transcription. Mol. Endocrinol 2005;19:2283-98. [PubMed: 15890677]

60. Matsuzaki H, Daitoku H, Hatta M, Aoyama H, Yoshimochi K, Fukamizu A. Acetylation of Foxo1 alters its DNA-binding ability and sensitivity to phosphorylation. Proc. Natl. Acad. Sci. USA 2005;102:11278-83. [PubMed: 16076959]

61. van der Horst A, Tertoolen LG, de Vries-Smits LM, Frye RA, Medema RH, Burgering BM. FOXO4 is acetylated upon peroxide stress and deacetylated by the longevity protein hSir2(SIRT1). J. Biol. Chem 2004;279:28873-79. [PubMed: 15126506] 
62. Ghosh HS. The anti-aging, metabolism potential of SIRT1. Curr. Opin. Investig. Drugs 2008;9:1095-102.

63. Tissenbaum HA, Guarente L. Increased dosage of a sir-2 gene extends lifespan in Caenorhabditis elegans. Nature 2001;410:227-30. [PubMed: 11242085]

64. Brunet A, Sweeney LB, Sturgill JF, Chua KF, Greer PL, et al. Stress-dependent regulation of FOXO transcription factors by the SIRT1 deacetylase. Science 2004;303:2011-15. [PubMed: 14976264]

65. Motta MC, Divecha N, Lemieux M, Kamel C, Chen D, et al. Mammalian SIRT1 represses forkhead transcription factors. Cell 2004;116:551-63. [PubMed: 14980222]

66. Daitoku H, Hatta M, Matsuzaki H, Aratani S, Ohshima T, et al. Silent information regulator 2 potentiates Foxo1-mediated transcription through its deacetylase activity. Proc. Natl. Acad. Sci. USA 2004;101:10042-47. [PubMed: 15220471]

67. Kitamura YI, Kitamura T, Kruse JP, Raum JC, Stein R, et al. FoxO1 protects against pancreatic $\beta$ cell failure through NeuroD and MafA induction. Cell Metab 2005;2:153-63. [PubMed: 16154098]

68. Plas DR, Thompson CB. Akt activation promotes degradation of tuberin and FOXO3a via the proteasome. J. Biol. Chem 2003;278:12361-66. [PubMed: 12517744]

69. Huang H, Regan KM, Wang F, Wang D, Smith DI, et al. Skp2 inhibits FOXO1 in tumor suppression through ubiquitin-mediated degradation. Proc. Natl. Acad. Sci. USA 2005;102:164954. [PubMed: 15668399]

70. Charvet C, Alberti I, Luciano F, Jacquel A, Bernard A, et al. Proteolytic regulation of Forkhead transcription factor FOXO3a by caspase-3-like proteases. Oncogene 2003;22:4557-68. [PubMed: 12881712]

71. Brenkman AB, de Keizer PL, van den Broek NJ, Jochemsen AG, Burgering BM. Mdm2 induces mono-ubiquitination of FOXO4. PLoS ONE 2008;3:e2819. [PubMed: 18665269]

72. van der Horst A, de Vries-Smits AM, Brenkman AB, van Triest MH, van den Broek N, et al. FOXO4 transcriptional activity is regulated by monoubiquitination and USP7/HAUSP. Nat. Cell Biol 2006;8:1064-73. [PubMed: 16964248]

73. Li HH, Willis MS, Lockyer P, Miller N, McDonough H, et al. Atrogin-1 inhibits Akt-dependent cardiac hypertrophy in mice via ubiquitin-dependent coactivation of Forkhead proteins. J. Clin. Investig 2007;117:3211-23. [PubMed: 17965779]

74. Hosaka T, Biggs WH 3rd, Tieu D, Boyer AD, Varki NM, et al. Disruption of forkhead transcription factor (FOXO) family members in mice reveals their functional diversification. Proc. Natl. Acad. Sci. USA 2004;101:2975-80. [PubMed: 14978268]

75. Furuyama T, Kitayama K, Shimoda Y, Ogawa M, Sone K, et al. Abnormal angiogenesis in Foxo1 (Fkhr)-deficient mice. J. Biol. Chem 2004;279:34741-49. [PubMed: 15184386]

76. Evans-Anderson HJ, Alfieri CM, Yutzey KE. Regulation of cardiomyocyte proliferation and myocardial growth during development by FOXO transcription factors. Circ. Res 2008;102:68694. [PubMed: 18218983]

77. Heinke J, Wehofsits L, Zhou Q, Zoeller C, Baar KM, et al. BMPER is an endothelial cell regulator and controls bone morphogenetic protein-4-dependent angiogenesis. Circ. Res 2008;103:804-12. [PubMed: 18787191]

78. Moser M, Binder O, Wu Y, Aitsebaomo J, Ren R, et al. BMPER, a novel endothelial cell precursor-derived protein, antagonizes bone morphogenetic protein signaling and endothelial cell differentiation. Mol. Cell. Biol 2003;23:5664-79. [PubMed: 12897139]

79. Potente M, Urbich C, Sasaki K, Hofmann WK, Heeschen C, et al. Involvement of Foxo transcription factors in angiogenesis and postnatal neovascularization. J. Clin. Investig 2005;115:2382-92. [PubMed: 16100571]

80. Potente M, Ghaeni L, Baldessari D, Mostoslavsky R, Rossig L, et al. SIRT1 controls endothelial angiogenic functions during vascular growth. Genes Dev 2007;21:2644-58. [PubMed: 17938244]

81. Creemers EE, Sutherland LB, McAnally J, Richardson JA, Olson EN. Myocardin is a direct transcriptional target of Mef2, Tead and Foxo proteins during cardiovascular development. Development 2006;133:4245-56. [PubMed: 17021041] 
82. Paik JH, Kollipara R, Chu G, Ji H, Xiao Y, et al. FoxOs are lineage-restricted redundant tumor suppressors and regulate endothelial cell homeostasis. Cell 2007;128:309-23. [PubMed: 17254969]

83. Shiojima I, Walsh K. Regulation of cardiac growth and coronary angiogenesis by the Akt/PKB signaling pathway. Genes Dev 2006;20:3347-65. [PubMed: 17182864]

84. Stitt TN, Drujan D, Clarke BA, Panaro F, Timofeyva Y, et al. The IGF-1/PI3K/Akt pathway prevents expression of muscle atrophy-induced ubiquitin ligases by inhibiting FOXO transcription factors. Mol. Cell 2004;14:395-403. [PubMed: 15125842]

85. Belke DD, Betuing S, Tuttle MJ, Graveleau C, Young ME, et al. Insulin signaling coordinately regulates cardiac size, metabolism, and contractile protein isoform expression. J. Clin. Investig 2002;109:629-39. [PubMed: 11877471]

86. Skurk C, Izumiya Y, Maatz H, Razeghi P, Shiojima I, et al. The FOXO3a transcription factor regulates cardiac myocyte size downstream of AKT signaling. J. Biol. Chem 2005;280:20814-23. [PubMed: 15781459]

87. Shioi T, McMullen JR, Kang PM, Douglas PS, Obata T, et al. Akt/protein kinase B promotes organ growth in transgenic mice. Mol. Cell. Biol 2002;22:2799-809. [PubMed: 11909972]

88. Ni YG, Berenji K, Wang N, Oh M, Sachan N, et al. Foxo transcription factors blunt cardiac hypertrophy by inhibiting calcineurin signaling. Circulation 2006;114:1159-68. [PubMed: 16952979]

89. Molkentin JD, Lu JR, Antos CL, Markham B, Richardson J, et al. A calcineurin-dependent transcriptional pathway for cardiac hypertrophy. Cell 1998;93:215-28. [PubMed: 9568714]

90. Li HH, Kedar V, Zhang C, McDonough H, Arya R, et al. Atrogin-1/muscle atrophy F-box inhibits calcineurin-dependent cardiac hypertrophy by participating in an SCF ubiquitin ligase complex. J. Clin. Investig 2004;114:1058-71. [PubMed: 15489953]

91. Ivaska J, Nissinen L, Immonen N, Eriksson JE, Kähäri VM, Heino J. Integrin $\alpha 2 \beta 1$ promotes activation of protein phosphatase $2 \mathrm{~A}$ and dephosphorylation of Akt and glycogen synthase kinase 3 3. Mol. Cell. Biol 2002;22:1352-59. [PubMed: 11839802]

92. Ni YG, Wang N, Cao DJ, Sachan N, Morris DJ, et al. FoxO transcription factors activate Akt and attenuate insulin signaling in heart by inhibiting protein phosphatases. Proc. Natl. Acad. Sci. USA 2007;104:20517-22. [PubMed: 18077353]

93. Wessells RJ, Fitzgerald E, Cypser JR, Tatar M, Bodmer R. Insulin regulation of heart function in aging fruit flies. Nat. Genet 2004;36:1275-81. [PubMed: 15565107]

94. Alcendor RR, Gao S, Zhai P, Zablocki D, Holle E, et al. Sirt1 regulates aging and resistance to oxidative stress in the heart. Circ. Res 2007;100:1512-21. [PubMed: 17446436]

95. Chen CJ, Yu W, Fu YC, Wang X, Li JL, Wang W. Resveratrol protects cardiomyocytes from hypoxia-induced apoptosis through the SIRT1-FoxO1 pathway. Biochem. Biophys. Res. Commun 2009;378:389-93. [PubMed: 19059213]

96. Chan AY, Dolinsky VW, Soltys CL, Viollet B, Baksh S, et al. Resveratrol inhibits cardiac hypertrophy via AMP-activated protein kinase and Akt. J. Biol. Chem 2008;283:24194-201. [PubMed: 18562309]

97. Chiribau CB, Cheng L, Cucoranu IC, Yu YS, Clempus RE, Sorescu D. FOXO3A regulates peroxiredoxin III expression in human cardiac fibroblasts. J. Biol. Chem 2008;283:8211-17. [PubMed: 18195003]

98. Abid MR, Guo S, Minami T, Spokes KC, Ueki K, et al. Vascular endothelial growth factor activates PI3K/Akt/forkhead signaling in endothelial cells. Arterioscler. Thromb. Vasc. Biol 2004;24:294-300. [PubMed: 14656735]

99. Abid MR, Spokes KC, Shih SC, Aird WC. NADPH oxidase activity selectively modulates vascular endothelial growth factor signaling pathways. J. Biol. Chem 2007;282:35373-85. [PubMed: 17908694]

100. Abid MR, Shih SC, Otu HH, Spokes KC, Okada Y, et al. A novel class of vascular endothelial growth factor-responsive genes that require forkhead activity for expression. J. Biol. Chem 2006;281:35544-53. [PubMed: 16980307] 
101. Allard D, Figg N, Bennett MR, Littlewood TD. Akt regulates the survival of vascular smooth muscle cells via inhibition of FoxO3a and GSK3. J. Biol. Chem 2008;283:19739-47. [PubMed: 18458087]

102. Chlench S, Mecha Disassa N, Hohberg M, Hoffmann C, Pohlkamp T, et al. Regulation of Foxo-1 and the angiopoietin-2/Tie2 system by shear stress. FEBS Lett 2007;581:673-80. [PubMed: 17258205]

103. Kim HS, Skurk C, Maatz H, Shiojima I, Ivashchenko Y, et al. Akt/FOXO3a signaling modulates the endothelial stress response through regulation of heat shock protein 70 expression. FASEB J 2005;19:1042-44. [PubMed: 15784720]

104. Gershman B, Puig O, Hang L, Peitzsch RM, Tatar M, Garofalo RS. High-resolution dynamics of the transcriptional response to nutrition in Drosophila: a key role for dFOXO. Physiol. Genomics 2007;29:24-34. [PubMed: 17090700]

105. Philip-Couderc P, Tavares NI, Roatti A, Lerch R, Montessuit C, Baertschi AJ. Forkhead transcription factors coordinate expression of myocardial KATP channel subunits and energy metabolism. Circ. Res 2008;102:e20-35. [PubMed: 18202312]

106. Daitoku H, Yamagata K, Matsuzaki H, Hatta M, Fukamizu A. Regulation of PGC-1 promoter activity by protein kinase B and the forkhead transcription factor FKHR. Diabetes 2003;52:64249. [PubMed: 12606503]

107. Zhang Y, Ma K, Sadana P, Chowdhury F, Gaillard S, et al. Estrogen-related receptors stimulate pyruvate dehydrogenase kinase isoform 4 gene expression. J. Biol. Chem 2006;281:39897-906. [PubMed: 17079227]

108. Yamada S, Kane GC, Behfar A, Liu XK, Dyer RB, et al. Protection conferred by myocardial ATP-sensitive $\mathrm{K}^{+}$channels in pressure overload-induced congestive heart failure revealed in KCNJ11 Kir6.2-null mutant. J. Physiol 2006;577:1053-65. [PubMed: 17038430]

109. Matsui Y, Kyoi S, Takagi H, Hsu CP, Hariharan N, et al. Molecular mechanisms and physiological significance of autophagy during myocardial ischemia and reperfusion. Autophagy 2008;4:409-15. [PubMed: 18227645]

110. Hamacher-Brady A, Brady NR, Gottlieb RA. Enhancing macroautophagy protects against ischemia/reperfusion injury in cardiac myocytes. J. Biol. Chem 2006;281:29776-87. [PubMed: 16882669]

111. Nakai A, Yamaguchi O, Takeda T, Higuchi Y, Hikoso S, et al. The role of autophagy in cardiomyocytes in the basal state and in response to hemodynamic stress. Nat. Med 2007;13:619-24. [PubMed: 17450150]

112. Zhao J, Brault JJ, Schild A, Cao P, Sandri M, et al. FoxO3 coordinately activates protein degradation by the autophagic/lysosomal and proteasomal pathways in atrophying muscle cells. Cell Metab 2007;6:472-83. [PubMed: 18054316] 
a

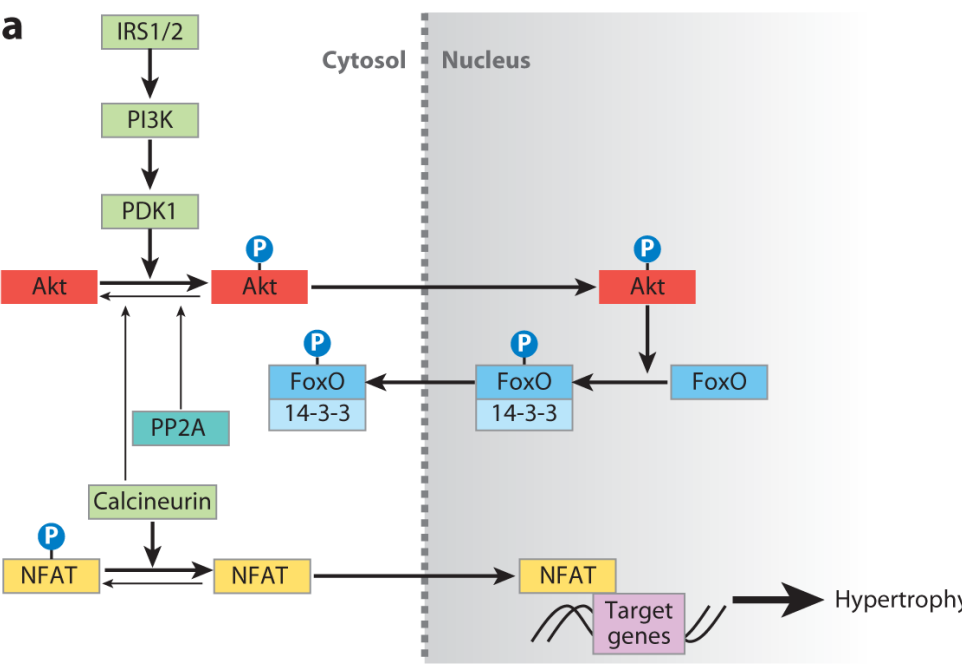

b

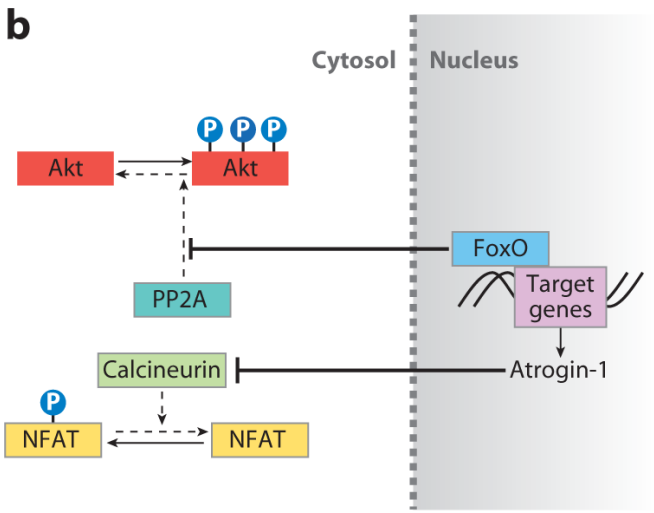

Figure 1.

Akt and FoxO signaling pathways in cardiac hypertrophy. (a) Insulin receptor signaling activates the PI3K (phosphoinositide-3 kinase) signaling cascade, leading to Akt phosphorylation and translocation into the nucleus. Akt phosphorylates three conserved FoxO residues to promote FoxO association with 14-3-3 proteins and nuclear export to the cytosol, where the complex remains transcriptionally inactive. Calcineurin dephosphorylates NFAT (nuclear factor of activated $\underline{T}$ cells), allowing NFAT nuclear translocation and subsequent transcription of NFAT target genes, such as those encoding $\alpha$-skeletal actin and $\beta$-myosin heavy chain. PP2A and calcineurin provide negative feedback by dephosphorylating Akt. (b) In the absence of PI3K signaling, FoxO is able to transcribe target genes such as atrogin-1, which encodes a ubiquitin ligase that promotes calcineurin degradation and prevents NFAT activation. The decrease in calcineurin levels reduces Aktassociated phosphatase activity. FoxO also reduces PP2A phosphatase activity on Akt. The reduction in calcineurin and $\mathrm{PP} 2 \mathrm{~A}$-associated phosphatase activity leads to Akt hyperphosphorylation, which eventually inhibits FoxO activity. 